\title{
Specifications and Standards for Insect 3D Data
}

\author{
Jiangning Wang ${ }^{\ddagger}$, Jing Ren ${ }^{\ddagger}$, Tianyu $\mathrm{Xi}^{\ddagger}$, Siqin $\mathrm{Ge}^{\ddagger}$, Liqiang $\mathrm{Ji}^{\ddagger}$ \\ ‡ Institute Of Zoology, Chinese Academy Of Sciences, Beijing, China
}

Corresponding author: Jiangning Wang (wangjn@ioz.ac.cn), Siqin Ge (gesiqin12@hotmail.com), Liqiang Ji (ji@i oz.ac.cn)

Received: 10 May 2018| Published: 21 May 2018

Citation: Wang J, Ren J, Xi T, Ge S, Ji L (2018) Specifications and Standards for Insect 3D Data. Biodiversity Information Science and Standards 2: e26561. https://doi.org/10.3897/biss.2.26561

\section{Abstract}

With the continuous development of imaging technology, the amount of insect 3D data is increasing, but research on data management is still virtually non-existent. This paper will discuss the specifications and standards relevant to the process of insect 3D data acquisition, processing and analysis.

The collection of 3D data of insects includes specimen collection, sample preparation, image scanning specifications and 3D model specification. The specimen collection information uses existing biodiversity information standards such as Darwin Core. However, the 3D scanning process contains unique specifications for specimen preparation, depending on the scanning equipment, to achieve the best imaging results.

Data processing of 3D images includes 3D reconstruction, tagging morphological structures (such as muscle and skeleton), and 3D model building. There are different algorithms in the $3 \mathrm{D}$ reconstruction process, but the processing results generally follow DICOM (Digital Imaging and Communications in Medicine) standards. There is no available standard for marking morphological structures, because this process is currently executed by individual researchers who create operational specifications according to their own needs. 3D models have specific file specifications, such as object files (https:// en.wikipedia.org/wiki/Wavefront .obj file) and 3D max format (https://en.wikipedia.org/

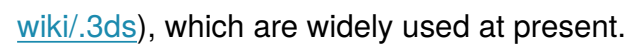


There are only some simple tools for analysis of three-dimensional data and there are no specific standards or specifications in Audubon Core (https://terms.tdwg.org/wiki/ Audubon Core), the TDWG standard for biodiversity-related multi-media.

There are very few 3D databases of animals at this time. Most of insect 3D data are created by individual entomologists and are not even stored in databases. Specifications for the management of insect 3D data need to be established step-by-step. Based on our attempt to construct a database of 3D insect data, we preliminarily discuss the necessary specifications.

\section{Keywords}

3D data standard; database; CT; insect

\section{Presenting author}

Jiangning Wang 